\title{
La phénoménologie à l'épreuve de la prière. Le nom de Dieu, et peut-être plus
}

Jérôme de Gramont

\section{(2) OpenEdition}

1 Journals

Édition électronique

URL : https://journals.openedition.org/alter/2036

DOI : 10.4000/alter.2036

ISSN : 2558-7927

Éditeur :

Association ALTER, Archives Husserl (CNRS-UMR 8547)

\section{Édition imprimée}

Date de publication : 1 novembre 2020

Pagination : 149-163

ISBN : 978-2-9550449-6-4

ISSN : $1249-8947$

\section{Référence électronique}

Jérôme de Gramont, «La phénoménologie à l'épreuve de la prière. Le nom de Dieu, et peut-être plus », Alter [En ligne], 28 | 2020, mis en ligne le 22 décembre 2020, consulté le 14 octobre 2021. URL : http:// journals.openedition.org/alter/2036; DOI : https://doi.org/10.4000/alter.2036

Ce document a été généré automatiquement le 14 octobre 2021

Revue Alter 


\title{
La phénoménologie à l'épreuve de la prière. Le nom de Dieu, et peut-être plus
}

\author{
Jérôme de Gramont
}

1 Au commencement de la vie religieuse, il pourrait y avoir un verbe humain fait de plus de doute que de savoir, de plus d'inévidence que d'évidence. Deux vers du poète Jules Supervielle portent jusqu'à une expression claire cette expérience encore obscure :

Voilà que je me surprends à t'adresser la parole,

Mon Dieu, moi qui ne sais encore si tu existes.

Ces deux vers constituent l'incipit d'un poème intitulé «Prière à l'inconnu » publié dans le recueil $L a$ Fable $d u$ monde ${ }^{1}$, et sont cités surtout au début de la petite «Phénoménologie de la prière » à laquelle Jean-Louis Chrétien donne pour titre « La parole blessée $\rrbracket^{2}$. Tout pourrait bien commencer par une voix nue et incertaine qui, avant même de savoir vers qui elle se porte, et avant même de se savoir altérée, blessée par son destinataire, cherche Dieu, ne trouvant encore que son nom, se surprenant à prononcer son nom, à lui adresser une parole. Seule sans doute une existence déjà blessée peut trouver en elle l'audace de parler ainsi à un Dieu inconnu.

Tout commencerait faiblement, dans une voix de peu de savoir, hormis un nom, juste un nom, celui qui vient aux lèvres ou au cœur, le nom de Dieu. Autrement dit, même si nous ignorons s'il existe, et même si tout de son être reste pour nous problématique, Dieu est le nom propre de celui vers qui se portent nos prières : celui à qui nous nous adressons en disant «Mon Dieu ! (Jean-Luc Nancy) ${ }^{3}$ ou celui que nous appelons quand nous disons «Viens! » (Jacques Derrida) ${ }^{4}$. Évidence et inévidence se mêlent dans cette parole - inévidence du côté de Dieu (puisque nous ne savons pas même si parler de Dieu lui-même a un sens), mais évidence qu'une prière a lieu, née de notre détresse d'homme, et cela même si personne ne vient répondre à cette parole que nous lançons loin de nous, au loin de nous, peut-être à vide («Supervielle, dans sa Prière à l'inconnu, parle à un Dieu dont il ne pose pas l'existence et dont il ignore s'il l'écoute $\left.»^{5}\right)$, même si notre intentionnalité porte à vide, comme pour Jupiter, lequel, pour reprendre un 
exemple célèbre de la Ve Recherche logique, s'il reçoit bien une "existence mentale " n'est douée d'aucune existence « extra mentem " ${ }^{6}$. De celui qui porte un nom puisque nos prières se portent vers lui, il est possible que nous ne sachions rien de plus, mais une parole s'est levée qui va au-devant de ce nom.

Or il faut maintenir le droit à celui qui n'a pas la foi d'adresser à Dieu une prière, et la possibilité de lire le poème de Supervielle comme plus que simplement un poème: comme une véritable prière. Un verbe sans assurance a ouvert l'espace de la prière, les premiers mots tournés vers Dieu auront été ceux d'un incroyant (d'un qui ne comprend pas la langue des églises ${ }^{7}$ ), car il faut du temps pour exister devant Dieu mais pas pour vivre dans le désarroi et appeler au secours. Celui qui ne sait rien de ce Dieu à qui il s'adresse, pas même s'il existe, ne le tutoie pas moins, et « cette prière à un Dieu virtuel [...] n'est pas elle-même une prière virtuelle, mais une prière effective et véritable $»^{8}$. Les mots que le poète se surprend à prononcer peuvent bien constituer une forme faible de la prière, ce qu'il faudrait même tenir pour sa forme la plus faible, cette faiblesse n'est pas étrangère à l'essence de la prière, sans quoi elle ne viendrait pas de nous. Celui qui ne sait pas prier et l'avoue a déjà commencé à prier. Mais qui peut dire qu'il connaît les mots justes lorsque ces mots doivent toucher Dieu?

Dans cette forme la plus faible de la prière, justement parce qu'elle atteste notre faiblesse et monte comme une plainte ( $" \mathrm{Et}$ au-dedans comme au dehors il y a la détresse humaine $»^{9}$ ), justement parce qu'elle prend élan dans le plus fragile en nous et cherche secours en Dieu, même s'il s'agit là d'un Dieu inconnu, il faut voir la vraie prière au moment où elle naît au cœur de l'homme. Comment les mots du poète ne retrouveraient-ils pas ceux des évangiles, car c'est pour le pécheur que s'ouvre l'espace du salut ( Je ne suis pas venu appeler les justes mais les pécheurs» Mc 2, 17) et pour celui qui est dans la détresse de vivre que s'ouvre l'espace de la religion. C'est d'être sauvés dont nous avons le plus besoin, et ce savoir est sans étude.

Évidence et inévidence se mêlent dans cette parole - évidence des mots que nous prononçons, mais inévidence de cette prière à l'inconnu. Inévidence de ce vers de Supervielle: «Mon Dieu, je ne crois pas en toi, je voudrais te parler tout de même ${ }^{10}$. Comment adresser une prière à celui dont on ne sait s'il peut l'entendre? Comment l'adresser sans au moins espérer qu'il puisse l'entendre ? Comment adresser à Dieu une prière sans lui tenir deux paroles, dont l'une est pour ce Dieu « sans visage et peut-être sans espérance $»^{11}$, et l'autre pour ce Dieu qui peut-être existe et dont nous espérons qu'il pourra nous répondre - car tout " peut-être » doit s'entendre deux fois, dans les possibilités adverses de ces deux fois.

7 Ces deux moments appartiennent au poème de Supervielle :

Je ne sais si tu entends nos prières, à nous les hommes,

Je ne sais si tu as envie de les écouter,

Si tu as comme nous un cœur qui est toujours sur le qui-vive,

Et des oreilles ouvertes aux nouvelles les plus différentes.

Je ne sais pas si tu aimes à regarder par ici,

Pourtant je voudrais te remettre en mémoire la planète Terre,

Avec ses fleurs, ses cailloux, ses jardins et ses maisons.

Avec tous les autres et nous qui savons bien que nous souffrons ${ }^{12}$.

8 Tout se retourne autour du mot "pourtant ", et ce qui commence dans la faiblesse et l'incertain ( «Je ne sais si tu entends nos prières ») devient la force de celui qui rappelle Dieu à son œuvre («je voudrais te remettre en mémoire la planète Terre », comme un appel à Dieu pour se rendre présent). Dans le poème de Supervielle nous pouvons 
entendre deux paroles, la première tenue dans la réserve d'un " peut-être » («Ah ! si tu existes, mon Dieu, regarde de notre côté $\left.{ }^{13} »\right)$, et la seconde à l'impératif («Mon Dieu, ne va pas faire encore la sourde oreille $\left.{ }^{14} »\right)$. Là où la prière se fait plus pressante, parce que l'histoire des hommes se fait plus menaçante, les mêmes mots peuvent résonner comme faiblesse ( je ne sais si mes mots peuvent t'atteindre ») ou comme force («tu dois m'écouter, écouter les souffrances de la terre et des hommes»). Tout se retourne autour du mot " pourtant ", comme une relance du possible là où il semblait épuisé, là où se présentait seulement un "Dieu atténué ", un Dieu sans puissance et pour cela déjà presque effacé, ou bien un "Dieu peut-être sans espérance ", mais où le mot peutêtre cette fois suffit pour indiquer que toute espérance n'est pas morte. Le possible renaîtrait là où les possibles semblent épuisés, son nom pourrait être l'impossible, celui qu'en tout cas lui donne Supervielle dans son poème :

Je veux t'adresser sans tarder ces humbles paroles humaines

Parce qu'il faut que chacun tente à présent tout l'impossible ${ }^{15}$.

Dieu apparaît maintenant comme le Dieu à l'impossible auquel nous adressons pourtant nos prières, comme un dernier recours au moment où tous les possibles de la terre sont épuisés - celui dont nous pouvons espérer qu'il soit et puisse nous venir en aide, d'une manière de nous inconnue. La prière adressée à un Dieu "sans visage et sans espérance » est adressée à Dieu dans l'espérance pourtant qu'il soit - même si cette adresse n'a lieu que dans l'horizon de l'espérance et jamais d'une évidence, et même si cette existence se voit marquée au sceau d'un peut-être. Celui qui se surprend à prier, ne sachant encore rien de celui à qui il s'adresse, ne peut s'empêcher pourtant d'espérer que sa prière soit entendue. Qu'elle soit seulement entendue, et sa prière aura commencé d'être exaucée.

Il y a dans cette «Prière à l'inconnu » un paradoxe qu'une phénoménologie de la prière, loin de dissiper, redouble. Une phénoménologie se doit de seulement décrire, mais une phénoménologie de la prière se voit tenue d'ajouter un mot à ses descriptions, au risque de franchir indûment une frontière dûment tracée par la méthode: celle de l'existence. Même si le phénoménologue par principe n'accomplit pas de position d'existence, la fidélité aux phénomènes qui est un principe encore plus fort pourrait bien nous y contraindre. Avançons quelques mots de plus à même le poème de Supervielle, et à ses deux premiers vers ajoutons-en librement un troisième :

Voilà que je me surprends à t'adresser la parole,

Mon Dieu, moi qui ne sais encore si tu existes,

Mais qui ne peux m'empêcher d'espérer que tu sois et que tu m'entendes.

11 Dès qu'il y a prière, il y a adresse à quelqu'un dont le nom propre est Dieu, dans l'espérance aussi que Dieu soit plus qu'un nom, autrement dit qu'il existe. Si une prière s'avance au-devant de Dieu (ou de son nom), elle ne peut faire l'économie de cette espérance que Dieu soit plus que son nom, et qu'il existe. Sinon cette parole qui le nomme et seulement le nomme se réduit (au sens trivial du vocable) à des mots, et elle n'est que littérature.

Cherchons un autre point de départ à nos considérations sur la prière. Nous le prendrons à Paul Celan et au poème célèbre et sur-commenté qu'il intitule « Psaume »un poème qui est aussi un authentique psaume et qui, même s'il ne s'adresse à personne, s'adresse encore à un tu. Rappelons quelques vers :

Loué sois-tu, Personne.

C'est pour toi que nous voulons

fleurir. 
A ta

rencontre ${ }^{16}$

13 Si le ton y est plus dramatique, nous retrouvons chez Celan les deux directions déjà indiquées à partir du poème de Supervielle : l'effacement du Dieu inconnu, l'espérance d'un Dieu encore possible. Et du poème de Celan trouvons un commentaire chez JeanYves Lacoste ${ }^{17}$. À nouveau deux paroles se font entendre dans le même poème, celle qui ne s'adresse à personne :

Non seulement il loue une absence radicale, ou un absent radical, mais il persiste à le tutoyer. Il le fait, d'autre part, après une expérience de décréation qui ne laisse ouvert aucun espoir de recréation. Le nihilisme, autrement dit, est accompli sans qu'une sortie hors du nihilisme semble offerte ${ }^{18}$.

et celle qui prend la figure d'un psaume :

De Celan, en revanche, il faut dire qu'il parle "malgré tout", ou malgré tout. [...] Le psaume est un genre littéraire biblique ou para-biblique. Celui qui, en des temps post-bibliques, donne le titre de "psaume" à un poème, ne choisit pas un mot innocent. Le psaume d'autre part, sous sa forme classique, est un texte liturgique, qui parle de et/ou à Dieu ${ }^{19}$.

15 C'est à cette tension, à l'arc produit par ces deux éléments : la prière à personne, et l'appel malgré tout à une rencontre, comme si le poème laissait s'élever une prière à Dieu qui dise : «Viens à ma rencontre, toi seul qui peut nous sauver du néant où nous sommes et où nous resterons peut-être ${ }^{20}$, que l'éros poétique se doit d'être à son état le plus haut, mais c'est aussi à cette tension que nous devons d'entrer dans l'espace des psaumes. Par une prière qui s'adresse à Dieu, comme toute prière, de demande comme de louange, mais qui vient de notre détresse, qui naît dans l'urgence d'un appel au secours lancé à un Dieu inconnu ou absent, dans l'espoir qu'il vienne à notre rencontre - aussi fait-elle figure de première prière, appelons-la la proto-prière ${ }^{21}$. Non pas une parole qui traverse l'espace entre terre et ciel, entre notre existence humaine et l'existence d'un Dieu que nous pourrions tutoyer parce qu'il nous fait face, mais cette traversée d'une parole impossible, lancée au moment où tous nos possibles humains sont épuisés, vers ce recommencement du possible: dans l'espérance ou la promesse que Dieu vienne jusqu'à nous. Qu'il vienne et pour cela qu'il soit. La prière, ou cette proto-prière, celle qui est lancée, il faudrait dire jetée, depuis le rien que nous sommes, ne pose pas l'existence du Dieu auquel elle s'adresse, elle s'adresse à lui, dans le battement d'un peut-être, entre l'impossible qui vient butter contre un Dieu inconnu ou absent, et le recommencement du possible qui ne viendra que de lui, du fait qu'il s'approche, qu'il vienne. Cette première prière n'affirme pas l'existence de celui à qui elle s'adresse, elle la désire, et sa première demande est pour que Dieu soit ${ }^{22}$ !

D'autres formes de prière pourront venir, elles ne sauraient effacer le droit de cette parole née dans l'urgence et le désir - l'urgence d'une existence, la nôtre, exposée à la nudité et la détresse, et le désir que Dieu soit plus qu'un nom et qu'il existe lui aussi, même si tout autrement - à se présenter comme la proto-prière. Cette parole a lieu, qu'il s'agit maintenant pour la phénoménologie de la prière de décrire, même si cet avènement constitue pour la phénoménologie elle-même un défi. En effet, cette parole n'a de sens que par l'intérêt qu'elle porte à l'existence de celui à qui elle s'adresse, là où la phénoménologie historique fait dépendre la tâche de constitution et donc tout son travail d'une réduction qui ne laisse rien indemne, ni le moi du philosophe ni Dieu. Nous sommes là sur un plan où la phénoménologie, si elle veut continuer à décrire les phénomènes, doit se porter à ses limites et accepter d'accomplir un pas au-delà de ces 
limites. La Phénoménalité de Dieu de Jean-Yves Lacoste est le livre qui met à son programme un tel pas.

Il y a peut-être des phénomènes que nous ne pouvons décrire sans admettre (préalablement ou dans le cours de la discussion) qu'ils ne peuvent être soumis à un travail de réduction (nous verrons qu'il y en a, et que ce sont probablement les phénomènes les plus intéressants ${ }^{23}$.

17 Tirons parti du travail lacostien, et gardons trois indications pour notre phénoménologie de la prière.

18 La première indication porte sur la parole la plus faible (la proto-prière) et une parole plus haute (celle du philosophe ou du théologien) que l'homme puisse tenir quand il s'agit de Dieu pour montrer qu'elles relèvent d'une même logique qui va à l'existence. Les présentes remarques sur le nom et plus-que-le-nom - sur l'existence en excès du nom - formulées à propos d'une prière naissante et incertaine (la « Prière à l'inconnu ») peuvent se répéter à propos de cette parole plus haute, cette forme à tout le moins plus construite de la parole qu'est le discours du penseur. Pour l'orant (même incroyant) comme pour le phénoménologue (même averti de l'impératif husserlien de réduction, celui de mettre hors-circuit toute position d'existence), a fortiori pour le théologien ${ }^{24}$, Dieu est l'irréductible vers lequel se porte notre parole, jusque dans ses aspects les plus variés (demande, confession de foi, louange). Lacoste aborde la question depuis la phénoménologie et ses contraintes: après avoir rappelé qu'en bonne orthodoxie husserlienne l'existence de Dieu tombe nécessairement sous le coup de la réduction, il peut bien ajouter qu'il est malgré tout de bonne phénoménologie, pour ne pas trahir la chose même, de reconnaître quand il le faut la réalité d'un irréductible, et ainsi de ne pas mettre hors-jeu la réalité de Dieu quand il s'agit de le décrire. Aussi les mêmes mots, la même question sur la réalité de Dieu pour moi (« Qui es-tu pour moi ? Qui suisje pour toi ?»), permettent-ils d'entrer en théologie et en phénoménologie :

C'est une bonne entrée en théologie. Et si c'est aussi de bonne phénoménologie, ce ne peut être qu'une phénoménologie qui reconnaît la réalité de l'irréductible (ici, celle du "tu") et se sait impuissante à mettre hors-jeu la réalité transcendante de ce qu'elle décrit. Une réduction qui se veut des ambitions universelles ne pourra les réaliser : celles-ci achopperont sur l'expérience de l'irréductible, ici sur celle d'un "tu", ou d'un "Tu" dont nous pouvons certainement faire un "il", mais dont nous ne pouvons certainement pas abandonner, ne serait-ce que par souci de méthode et par fidélité au "métier" de philosophe, la croyance qu'il existe hors du champ de notre conscience ${ }^{25}$.

19 Ici et là, dans la parole de l'orant comme dans le discours du pen-seur, avec des tons de voix assurément différents, une parole s'adresse à un «tu ». Comment pourrait-elle rester indifférente à son existence ou son inexistence ? Certes il y a loin entre la forme la plus faible de la prière (la prière à l'inconnu, aux limites de la croyance) et sa forme la plus haute (la confession de foi ou la louange), comme il y a loin entre la prière de Supervielle ou le psaume de Celan et les prières d'un Augustin («qui parle de Dieu en parlant à Dieu ») ou d'un Anselme (qui « insère un argument philosophico-théologique à l'intérieur d'une prière $»^{26}$ ). Mais ces différences n'effaceront pas ce qui est commun à tous ces actes de parole: nous ne pouvons ni parler de Dieu ni parler à Dieu en commençant par le mettre hors-jeu. Ce qu'il faut affirmer pour la foi, et pour la phénoménologie dès lors qu'elle veut dire quelque chose de Dieu en restant fidèle à sa phénoménalité propre :

Nous ne pouvons parler de Dieu rigoureusement sans rendre justice à une phénoménalité qui interdit toute réduction. [...] La foi, est-il nécessaire d'y insister, 
a "besoin" que Dieu existe afin de parler de lui. Nulle réduction spontanée ne peut trouver ici un logement. Et plus encore: une réduction méthodologique serait illégitime, elle le serait parce qu'elle se méprendrait sur un mode d'apparaître, son travail serait donc celui d'une phénoménologie infidèle à son propos ${ }^{27}$. plus sentir que dans l'urgence de ces prières qui sont autant d'appels à Dieu du fond de notre détresse (la prière de Supervielle, le psaume de Celan)? Nous n'avons pas moins besoin que Dieu existe pour le prier que pour le penser, et la leçon lacostienne vaut autant quand il s'agit de parler à Dieu que lorsqu'il s'agit de parler de lui : «Si nous décidions que nous n'avons pas "besoin" que le Dieu dont nous parlons soit un Dieu auquel nous parlons, l'expérience sur laquelle nous fonderions ce que nous dirions de Dieu serait plus que suspecte $\aleph^{28}$. pouvons y prendre. L'indication paraîtra d'autant plus forte qu'elle passera par le détour de la pensée, ou de notre existence il ne devrait tout simplement pas être question. Il y a une logique de la philosophie qui s'exerce sur la phénoménologie en tant que logos, et qui au nom d'une recherche du savoir commande de mettre hors-jeu le moi du penseur. Au regard de cette logique notre existence singulière ne peut faire figure que de quantité négligeable et sacrifiable. Cette logique du désintéressement ne manque de trouver dans la phénoménologie husserlienne ses lettres de noblesse, mais elle se heurte à nouveau à certains phénomènes qui demandent de lui faire exception. Nous lisons au $§ 8$ des Thèses sur le vrai de Jean-Yves Lacoste cette double affirmation qui vise directement Husserl :

Il faut savoir occuper la situation du spectateur désintéressé, sans laquelle nous ne pourrions pas fonder notre recours à l'évidence - sans laquelle nous ne pourrions prouver notre droit à parler d'évidence. Or, il faut aussi apprendre à quitter cette situation, ou plus exactement savoir qu'il s'agit là d'une position occupée d'une manière aussi provisoire qu'indispensable ${ }^{29}$.

Aucune distance prise à l'égard de sa prière n'a de sens pour l'homme qui prie, pas même pour amener à l'évidence son attitude devant Dieu. L'homme qui prie ne peut pas se regarder prier : il prie, avec ses mots et son corps - homme réel, en chair et en os, campé sur une terre qui fait face au ciel, présent là devant Dieu de toute sa présence la plus concrète, la plus physique, la plus charnelle, de toute son humanité donc ${ }^{30}$. C'est l'homme qui prie et pas la conscience - dire autre chose, détacher la prière de ce poids d'humanité pour la livrer en spectacle à un sujet désintéressé, reviendrait à vider de son sens le phénomène de la prière. «Ce n'est pas un sujet, ou une âme, ou un intellect, qui se tient liturgiquement coram Deo, mais un homme en chair et en os, et dont le corps lui-même s'affronte à l'Absolu ${ }^{31}$.

Or nous connaissons un penseur dans l'histoire - qui ne se disait pas philosophe, n'avait pas l'autorité du théologien, préférait s'appeler poète du christianisme que chrétien et qui nous a appris la nécessité de quitter cette logique du concept, de l'abstraction et du système au nom de l'intérêt que l'existant prend à lui-même. Kierkegaard nous a appris à nous défier du langage du désintéressement, et qu'il n'y a aucune faute à nous intéresser à notre propre existence, même si c'est Johannes Climacus qui l'écrit de la manière la plus claire dans le Post-scriptum aux Miettes philosophiques: "L'intérêt suprême pour l'existant est d'exister, et l'intérêt qu'il porte au fait d'exister est la réalité $»^{32}$. À l'époque le combat avec la pensée théorique qui n'est que théorique est un combat avec Hegel, mais c'est bien dans un livre de phénoménologie, au $\$ 22$ des Thèses 
sur le vrai, que Jean-Yves Lacoste en fait mémoire : il y a un pouvoir de divertir dans les théories qui ne sont que théories « et qui, ce faisant, nous désintéressent de nousmêmes en notre insubstituable unicité $»^{33}$. Assurément la phénoménologie peut prolonger le rêve de philosophie comme science rigoureuse, une rigueur qui oblige à réduire "ce qui est mien et ce qui n'est que mien $»^{34}$, mais elle peut aussi «s'intéresser à l'unique événement dans lequel le soi se construit $»^{35}$.

La prière met en jeu un existant et l'Absolu, pour qu'un individu (un unique) entre dans un rapport absolu avec l'Absolu - non pas avec un Dieu, mais avec le Dieu d'Abraham, d'Isaac et de Jacob, ou pour le dire encore autrement, pour qu'un « je » et un «tu » viennent à se rencontrer. Or dans cette rencontre où il est question de se tenir devant Dieu, coram Deo, il s'agit bien aussi de notre présence. Celui qui dans la prière s'expose en présence de Dieu vient aussi à lui-même. Celui qui tutoie le Dieu auquel il s'adresse s'éprouve également en retour comme un unique capable de dire "je». Lorsque Kierkegaard, dans un chapitre des Euvres de l'amour pourtant intitulé "L'amour ne cherche pas son intérêt ", parle du fait "d'être soi-même devant Dieu ", il peut bien placer l'accent sur ce " devant Dieu », il n'en ajoute pas moins : « c'est en effet de cette attitude que découle tout le caractère de la personne ${ }^{36}$. Comprenons ainsi : l'amour ne cherche pas d'abord son intérêt, mais il le trouve de surcroît. Celui qui se tient devant Dieu découvre aussi ce que veut dire être soi, c'est-à-dire ici d'être cet unique en train de prier. Ce que Jean-Louis Chrétien, dans une étude sur «La prière selon Kierkegaard", commente ainsi: "La prière est aussi le lieu de l'individuation spirituelle $»^{37}$. Et Jean-Yves Lacoste, mais hors toute référence directe au penseur danois : «La prière est une preuve de l'existence de l'homme " $^{38}$.

La troisième indication lacostienne porte sur la relation entre l'homme en prière et Dieu, pensée du côté de Dieu comme modalité de sa présence et de notre côté comme modalité de la parole. Or si de part et d'autre nous avons à imaginer ou définir une forme haute (de présence ou de parole), nulle part celle-ci ne saurait passer pour la seule possible. Présence de Dieu - nous l'imaginons sans défaut possible, venant de Dieu, mais est-ce ainsi que le vit l'homme qui prie? A cela il faut répondre : non, et se rendre à l'évidence de cette inévidence. Même si la promesse de Dieu ne cesse jamais, ce n'est pas ainsi qu'a lieu la rencontre de l'homme avec Dieu - rencontre possible, et pour cela même adossée à la possibilité adverse d'une absence. Ce qui se livre alors comme une rencontre possible n'a pas lieu autrement que sous la modalité d'un peutêtre, comme ce qui peut venir ou ne pas venir, dans l'attente de celui dont nous ne savons pas s'il viendra, dont la présence peut faire défaut plutôt, elle qui ne s'atteste pas dans notre expérience. Un espace s'est ouvert, mais qui ne suffit pas pour que la venue de l'Absolu soit manifeste.

Celui qui prie n'entre pas en prière comme en un champ d'expérience où les données de la conscience mettraient évidemment l'Absolu en cause, mais ne fait qu'y ménager l'espace d'une possibilité - car il peut très bien ne rien se passer dans la liturgie qui soit l'incontestable avent de Dieu [...], car la liturgie peut fort bien, au plus profond d'elle-même, se présenter comme non-événement ${ }^{39}$.

Songeons-y, une phénoménologie de l'événement ne voudrait rien dire si elle ne laissait place à cette autre possibilité, du non-événement, celle qu'un espace s'ouvre et un temps s'écoule sans que rien d'absolument nouveau ne vienne. Il n'y a d'événement possible que là où il peut aussi n'y avoir que du vide, et seul vient à notre rencontre celui qui peut ne pas venir - ce serait sinon imaginer un Dieu disponible, et ce ne serait pas $\mathrm{Dieu}^{40}$. Celui qui prie est sans assurance que l'Absolu soit là et fasse sentir sa 
présence. Après la prière à l'inconnu (Supervielle) ou le psaume à personne (Celan), faudrait-il décrire une prière à l'Absent?

Dieu donc, si l'on décline ici l'offre qui nous est faite de ne penser sa possible présence que comme assomption d'un être-là ou comme participation à la logique de l'être-là, devrait-il être pensé comme absent ? Serait-il l'Absent au même titre qu'il lui arrive d'être dit Autre? Et faudrait-il alors entendre la liturgie comme espoir que l'absence présente de Dieu puisse "un jour" être comblée ${ }^{41}$ ?

Celui qui prie ne s'adresse pas à Dieu sans espérer qu'il vienne, mais il ne peut forcer l'Absolu à être là et faire sentir sa présence, il ne peut contraindre à venir celui qui peut ne pas venir (comme notre expérience tant de fois le montre, et comme Jean-Yves Lacoste peut l'écrire dans une simple parenthèse: "l'Absolu peut ne pas venir à l'expérience de la conscience, ce dont on conviendra que c'est le pain quotidien de la prière $\aleph^{42}$ ), il ne peut empêcher sa présence d'être toujours marquée au sceau d'une incontournable équivoque (peut-être une présence et peut-être une absence, peut-être que rien n'a eu lieu ou sous couvert seulement d'une équivoque ${ }^{43}$ ). Au moins peut-il toujours espérer une présence, et même une toute présence qui ne lui sera pas octroyée. Non que Dieu ne puisse venir à lui, mais seulement dans le clair-obscur du monde, et selon une modalité de la présence qui diffère d'avec la plénitude de la parousie. De là ces deux affirmations de la phénoménologie lacostienne de la liturgie : de notre côté l'espérance que Dieu vienne et réponde à notre prière, et du côté de Dieu un mode de présence qui diffère à la fois de la présence propre aux objets mondains et de la «toute présence eschatologique » (celle que le théologien nomme parousie). «On qualifiera donc d'abord la liturgie comme attente ou désir de la parousie dans la certitude de la présence non parousiaque de Dieu $»^{44}$. De là pour notre phénoménologie de la prière cette demande que Dieu vienne, de quelque manière ensuite qu'il nous réponde - cet appel qui se ramasse en un mot, le même que prononçait déjà la « Prière à l'inconnu » $^{45}$ et devinait le "Psaume » à personne : "Viens!». Même imprononcé, le premier mot de la première prière. "L'homme qui prie confesse son historialité et appelle l'eschaton en demandant que "vienne" le Dieu déjà présent dans l'ordre clairobscur du monde $»^{46}$.

Il reste que ce premier mot, encore incertain, ne sachant à qui il s'adresse, ou ne sachant si viendra celui à qui il s'adresse, n'épuise pas l'espace de la prière. D'autres mots peuvent être prononcés, d'autres paroles s'avancer, et l'homme qui prie peut exister devant Dieu selon d'autres dispositions que le désarroi de celui qui appelle depuis sa misère d'homme. D'autres formes de prière demandent à être décrites et d'abord, à l'autre extrémité de ce prisme des paroles adressées à Dieu, l'action de grâce ou la louange. Nous n'existons pas dans le monde selon le seul mode de l'angoisse, et nous ne vivons pas dans l'histoire selon le seul mode de la détresse. Il est heureux que nous puissions connaître d'autres tonalités affectives et qu'une joie toujours possible soit détentrice aussi d'un "sens de l'être ». Cela est heureux, et cela aussi demande à être porté au langage, dans une parole qui soit de gratitude et de bénédiction ${ }^{47}$. Nous pouvons faire monter vers Dieu nos plaintes d'homme, comme nous pouvons lui adresser des paroles de louange.

29 Assurément la distance est grande entre ces deux formes de prière, et comme souvent noter une différence revient à reconnaître une hiérarchie. À bien des titres la louange peut passer pour la prière par excellence - Jean-Yves Lacoste la présente dans Expérience et Absolu comme l'essence de la prière ${ }^{48}$, et Jean-Louis Chrétien citant Kierkegaard comme son accomplissement ${ }^{49}$. À la prière de louange donc le prestige de 
la contemplation, à celle de demande la moindre valeur d'une parole avant tout intéressée. Cette différence indéniable ne saurait pourtant conduire à disqualifier la première prière et nier son lien avec la louange. C'est de plusieurs manières que nous prions, mais c'est bien le même homme qui prie et s'efforce de se tenir devant Dieu, comme c'est le même pouvoir de la parole qui se déploie depuis les deux manières de faire résonner la même adresse : «Mon Dieu!", tantôt comme un appel («Viens à mon secours! ») et tantôt comme une louange (« Loué sois-tu!»). Une même voix se montre capable de ces deux tons de voix qu'il faut se garder de trop vite séparer. Plainte et louange sont liées, indissociables, chez celui qui plongé dans les remous d'histoire cherche à les traverser et se tourne vers celui qui domine l'histoire. Ces lignes de JeanYves Lacoste le disent :

Il va de soi que la parole est phénomène historial : nous ne pouvons décrire une parole extérieure au monde. Il va presque de soi, d'autre part, que la parole liturgique prétend échapper au gouvernement de l'histoire, et que l'homme qui rend grâce veut joindre sa voix aux chœurs angéliques. La doxologie a peut-être un avenir éternel. Le lien existant qu'elle entretient avec l'urgence des demandes révèle néanmoins qu'elle n'anticipe jamais cet avenir éternel qu'en avouant son historialité. La face historiale et la face (pré)eschatologique de la parole liturgique sont à prendre ensemble ou à laisser ensemble ${ }^{50}$.

et celles-ci de Jean-Louis Chrétien ne disent rien d'autre :

La prière de louange est l'essence même de la prière, ce dont toute autre prière provient et ce vers quoi elle tend. Un dieu qu'on ne pourrait pas remercier est un dieu à qui l'on ne pourrait rien demander, que l'on ne pourrait d'aucune façon prier $^{51}$.

Un même espace de parole réunit le bas et le haut, la plainte de la terre et le chant du ciel, le commencement et l'eschaton, la plus faible prière (la proto-prière), parole risquée au milieu des urgences d'histoire, et cette plus haute parole qui nomme le Dieu Seigneur de l'Être et de l'histoire, et lui rend gloire - comment parler sinon d'espace, comment imaginer une histoire pour l'homme en prière?

Deux choses, au moins, seraient alors à montrer. Comment supplication et doxologie constituent les deux faces de la prière, d'une parole où se dit quelque chose de nous (de notre faiblesse ou détresse) et quelque chose de Dieu (dans la mesure où l'homme qui prie reconnaît que " toute grâce excellente et tout don parfait descendent d'en haut ", Jc 1,17$)^{52}$. Deux faces, impossibles à confondre, mais qu'il ne faut pas séparer, d'une même parole où chaque versant rend possible l'autre, comme Jean-Louis Chrétien aura commencé de le dire dans L'Arche de la parole: la plainte parce que toute prière, même de louange, prend naissance dans notre condition et atteste notre misère (« Pour s'en tenir à la prière au Dieu unique, celui, quel qu'il soit, qui s'adresse à Dieu toujours le fait de profundis, des profondeurs de sa détresse manifeste ou cachée, des profondeurs de son péché $»^{53}$ ) et la louange parce qu'elle seule peut donner sens à nos larmes et nos cris, et permet de traverser notre possible détresse («Seule la louange peut rendre possible la plainte, car seul l'amour peut vraiment souffrir. N'y aurait-il rien au monde dont nous puissions rendre grâces qu'une plainte ne formerait qu'une creuse vocifération ${ }^{54}$ ). Comment surtout la prière nous fait entrer dans un mouvement qui prend naissance dans la plainte (la proto-prière comme adresse incertaine) et, parce qu'elle pose Dieu comme le seul capable de répondre à notre appel et l'origine de tout bien, s'achemine vers la louange et l'action de grâce. Ce mouvement qui porte la prière de sa forme la plus faible vers son accomplissement et sa forme la plus haute, de louange ${ }^{55}$. 
chose encore est à rappeler: l'éminente dignité des commencements même humbles, et qu'il n'est pas de prière inchoative qui ne soit déjà tout entière prière. L'homme qui simplement prie et élève vers un Dieu encore sans visage et dont il ne connaît que le nom («Mon Dieu!») une prière incertaine ne demande ni au philosophe ni au théologien, s'il a droit d'entrer dans cet espace de parole où il espère n'être pas seul. Il jette ses mots en avant, et son existence entière avec eux, sans savoir encore s'il atteindra l'autre rive.

\section{NOTES}

1. J. Supervielle, La Fable du monde, suivi de Oublieuse mémoire, Paris, Gallimard, 1987, p. 47.

2. J.-L. Chrétien, L'Arche de la parole, Paris, PUF, 1998, p. 23-54.

3. Voir J.-L. Nancy, « Mon Dieu! » dans Dieu en tant que Dieu. La question philosophique, P. CapelleDumont (éd.), Paris, Cerf, 2012 ( «je propose “mon Dieu !” comme une énonciation plus juste du nom "Dieu", le "mon" étant d'abord chargé de simplement noter l'exclamation et l'adresse... » p. 272).

4. Pour une interprétation de Dieu comme destinataire de cet appel, voir notre commentaire de Jacques Derrida (D'un ton apocalyptique adopté naguère en philosophie, Paris, Galilée, 1983) dans «Marges de la voix » (Bologne, Divus Thomas, 2019/1).

5. J.-L. Chrétien, L'Arche de la parole, op. cit., p. 23.

6. Voir E. Husserl, Recherches logiques, Hua XIX,1, p. 386 sq ; trad. par H. Elie, A. Kelkel, R. Schérer, tome II/2, Paris, PUF, 1961, p. 175.

7. Comme l'avoue le troisième vers du poème : «Et ne comprends pas la langue de tes églises chuchotantes » (J. Supervielle, op. cit. p.47).

8. J.-L. Chrétien, L'Arche de la parole, op. cit., p. 23 sq.

9. J. Supervielle, La Fable du monde, op. cit., p. 47.

10. Ibid., p. 48.

11. «Je voudrais, mon Dieu sans visage et peut-être sans espérance » (ibid., p. 49).

12. Ibid., p. 48.

13. Ibid., p. 50.

14. Ibid., p. 50.

15. Ibid., p. 48.

16. Trad. par J.-P. Lefebvre, dans Anthologie bilingue de la poésie allemande, Paris, Gallimard, «Pléiade ", 1993, p. 1189. Même si Jean-Louis Chrétien ne commente pas directement " Psaume », nous pouvons lire comme un commentaire implicite ses lignes à propos d'un autre poème de Celan, « Tenebrae » dans L'Antiphonaire de la nuit, Paris, L'Herne, 1989, p. 46.

17. J.-Y. Lacoste, Recherches sur la parole, Louvain, Peeters, 2015, p. 264-266.

18. Ibid., p. 265.

19. Ibid. p. 265.

20. «Un rien, voilà ce que nous fûmes, sommes et / resterons, fleurissant » (Paul Celan, «Psaume »).

21. Comme exemple de première prière (ou de ce qu'il nomme une «première parole dans [une] prière première ", Jérôme Thélot donne la demande : «Donnez-moi mon pain quotidien » $(\mathrm{Au}$ commencement était la faim. Traité de l'intraitable, La Versanne, Encre marine, 2005, p. 158 sq.), et 
ajoute: "L'athée lui-même, dans cette épreuve du pourquoi absolu, dans cette souffrance primordiale de la vie à mort, ne peut pas ne pas invoquer Dieu » (p. 162).

22. La première prière est pour cette demande paradoxale que nous pouvons lire chez Albert Cohen, dans un moment où il sent sa fin proche : «Viens à moi, prouve-moi que Tu n'es pas que dans mon imagination, prouve-moi que Tu es. Je veux croire, de toute mon âme croire. ô Dieu, mon amour, sois enfin merveilleusement Celui en qui je puisse croire" (Carnets 1978, Paris, Gallimard, 1979, p. 186).

23. J.-Y. Lacoste, La Phénoménalité de Dieu, III étude («L'apparaître et l'irréductible »), Paris, Cerf, 2008 , p. 58.

24. «[...] le théologien ne se demande pas si Dieu existe. La théologie est descriptive (ou narrative, les deux se recoupent largement ici) de part en part. Mais elle est descriptive à l'intérieur d'un acte de croyance » (ibid., p. 78 sq.).

25. Ibid., p. 78.

26. Ibid., p. 79 pour les deux références.

27. Ibid.p. 79.

28. Ibid., p. 85.

29. J.-Y. Lacoste, Thèses sur le vrai, Paris, PUF, 2018, p. 66.

30. De toute son humanité et de tout son poids, celui qui s'éprouve dans la fatigue. Sur cette prière par temps de fatigue voir J.-Y. Lacoste, Présence et parousie, Genève, Ad Solem, 2006, p. 314 : «Alourdis par notre corps et notre esprit, embarrassés par ce qui nous interdit de transgresser les limites mondaines de l'existence, les créatures fatiguées que nous sommes prient mal, et doivent le savoir ».

31. Ibid., p. 134.

32. S. Kierkegaard, Post-scriptum..., trad. par P.-H. Tisseau et E.-M. Jacquet-Tisseau, Euvres complètes, t. XI, Paris, L'Orante, 1977, p. 14.

33. J.-Y. Lacoste, Thèses sur le vrai, op. cit., p. 154.

34. Ibid., p. 154.

35. Selon une expression à la VIII étude de La Phénoménalité de Dieu, « De soi à soi. Présent vivant à et avenir infini », p. 194.

36. S. Kierkegaard, Les CEuvres de l'amour, trad. par P.-H. Tisseau et E.-M. Jacquet-Tisseau, CEuvres Complètes, t. XIV, Paris, L'Orante, 1990, p. 251.

37. J.-L. Chrétien, Le Regard de l'amour, Paris, Desclée de Brouwer, 2000, p. 110.

38. J.-Y. Lacoste, « Bâtir, habiter, prier » (II), Revue thomiste, octobre 1987, p. 561.

39. J.-L. Expérience et Absolu. Questions disputées sur l'humanité de l'homme, Paris, PUF, 1994, p. 56.

40. Nous n'appelons pas Dieu comme nous appelons les objets à paraître : «face à un Absolu auquel il reconnaît sa liberté, l'homme qui prie apprend [...] [que] son attente de Dieu ne contraindra jamais la condescendance de Dieu » (ibid., p. 57).

41. Ibid., p. 55.

42. Ibid., p. 58. Même expression dans Présence et parousie, op. cit., p. 315 sq. : « l'expérience de la fatigue est le pain quotidien de la prière ».

43. «[...] rien peut-être n'adviendra qui, dans la sphère d'immanence de la conscience, rende à la condescendance de Dieu un témoignage libre d'équivoque " (Expérience et Absolu, op. cit., p. 57).

44. J.-Y. Lacoste, Expérience et Absolu, op. cit., p. 55.

45. "Ah si tu existes, mon Dieu, regarde de notre côté, / Viens te délasser parmi nous " (J. Supervielle, La Fable du monde, op. cit., p. 50).

46. J.-Y. Lacoste, Présence et parousie, op. cit., p. 56.

47. Voir J.-Y. Lacoste, Être en danger, Paris, Cerf, 2011, p. 199.

48. «Nous prions certes pour louer - c'est en tout cas dans la louange que la prière manifeste purement son essence -, et la louange peut passer à bon droit pour l'image ou l'inchoation historique d'une pratique eschatologique » (J.-Y. Lacoste, Expérience et Absolu, op. cit., p. 52). 
49. «La doxologie ne pourrait être la conclusion de la prière des prières si toute prière chrétienne n'était originellement tendue vers elle comme vers son accomplissement » (J.-L. Chrétien, Le Regard de l'amour, op. cit., p. 117 sq., après avoir cité Kierkegaard OC XVI, 330).

50. J.-Y. Lacoste, Recherches sur la parole, op. cit., p. 238 sq.

51. J.-L. Chrétien, Le Regard de l'amour, op. cit., p. 118.

52. Sur ces deux faces de la prière, voir L'Arche de la parole, p. 30, et p. 40 cette belle formule : «L'adoration de l'Esprit qui fait crier a lieu dans le cri même ».

53. Ibid., p. 33.

54. Ibid., p. 197 - avec cette citation de Rilke, prise aux Sonnets à Orphée (I, 8) : «Ce n'est que dans l'espace de la louange que la plainte peut venir». Même idée chez Jérôme Thélot, $A u$ commencement était la faim, op. cit., p. 165 : «Avant la prière première, il y a, par prescription d'essence, une gratitude immémoriale qui la rend possible. Avant l'imprécation et la supplication, il y a leur condition transcendantale dans la louange. »

55. Ce sont là pages difficiles à écrire, mais pages déjà écrites par Ricœur dans son beau commentaire du Psaume 22, quand il décrit précisément le renversement de la plainte en louange - voir P. Ricœur et André LaCocque, Penser la Bible, Paris, Seuil, 1998, p. 286-289. 